\title{
QUANTITATIVE ELECTROMYOGRAPHIC CHANGES ASSOCIATED WITH MUSCULAR WEAKNESS
}

\author{
BY \\ J. A. R. LENMAN* \\ From the Neurological Unit, the Northern General Hospital, Edinburgh
}

It has been shown that when a human muscle contracts the mean voltage of the electromyogram recorded from its surface increases with the force of the contraction (Bayer and Flechtenmacher, 1950; Inman, Ralston, Saunders, Feinstein, and Wright, 1952; Lippold, 1952). The relationship between tension and mean voltage is linear provided the contraction is isometric (Lippold, 1952). With an isotonic contraction the voltage depends also on the velocity of shortening of the muscle (Bigland and Lippold, 1954) so that the relationship only remains linear if that is held constant. If a muscle is fatigued by a prolonged isometric contraction the relationship between voltage and tension remains linear but is of a different slope. More electrical activity is associated with a given tension in the fatigued state (Edwards and Lippold, 1956). This is also true if the fatigue results from isotonic exercise (Scherrer, Samson, and Paléologue, 1954).

Under standardized conditions it is possible to record similar voltage/tension curves from the same subject on different occasions (Lenman, 1959). The experiments described here were carried out to determine the range of slope for the curves relating mean voltage and tension in the upper limb muscles of healthy subjects and of patients with weak muscles resulting from neurological or muscular disease.

\section{Material and Methods}

Simultaneous records were made of the isometric tension and the mean voltage recorded from surface electrodes over the biceps or triceps muscle of 12 healthy subjects and 26 patients with weakness of these muscles. The measurements were made by the method described before (Lenman, 1959). The tension was recorded by a strain gauge dynamometer in which the gauges formed part of a Wheatstone bridge fed by alternating current. The out-of-balance voltage, which was proportional to the applied force, was amplified and appeared on one channel of a pen recorder. The electromyogram was recorded from surface electrodes of $1 \mathrm{~cm}$. diameter

* Present address: The Department of Medicine, Queen's College, Dundee. placed $3 \mathrm{~cm}$. apart over the belly of the muscle. It was amplified and fed into an integrator circuit (Bates and Cooper, 1954) producing pulses of frequency proportional to the mean voltage. These appeared on the other channel of the pen recorder.

Curves relating tension (lb.) to mean voltage (mV) were made by recording these simultaneously while the subject carried out a series of isometric contractions at different tensions, the apparatus being calibrated as described before (Lenman, 1959). The ratio tension/ electrical activity may be looked on as a measure of neuromuscular efficiency. In this presentation the reciprocal (voltage/tension) has been used so that the results can be compared with those of Lippold (1952).

\section{Results}

Experiments on Healthy Subjects.-The tension of the biceps or triceps was recorded simultaneously with the integrated electromyogram throughout a range of contractions in 12 healthy subjects. The mean values over five-second epochs were deter-? mined and voltage/tension curves constructed. The regression equation $(\mathrm{Y}=\mathrm{a}+\mathrm{bx})$ for each curve was calculated and the slope (b) was expressed as a regression coefficient (Fig. 1).

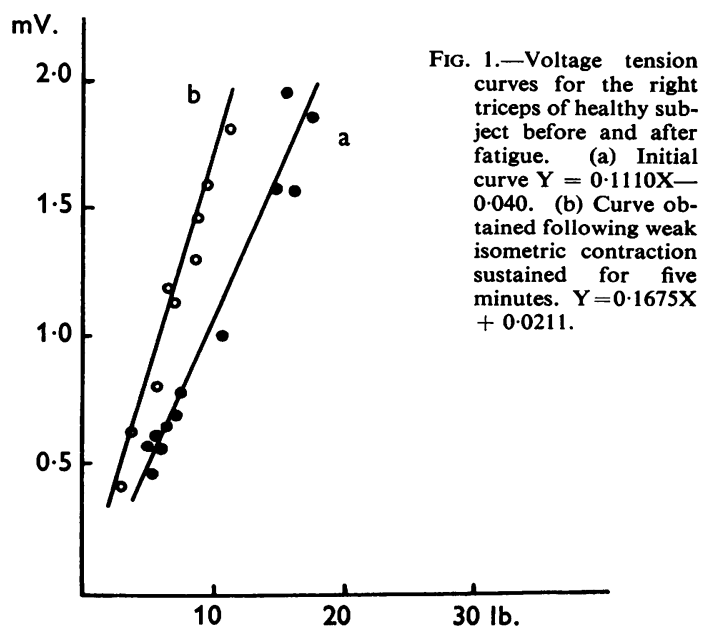




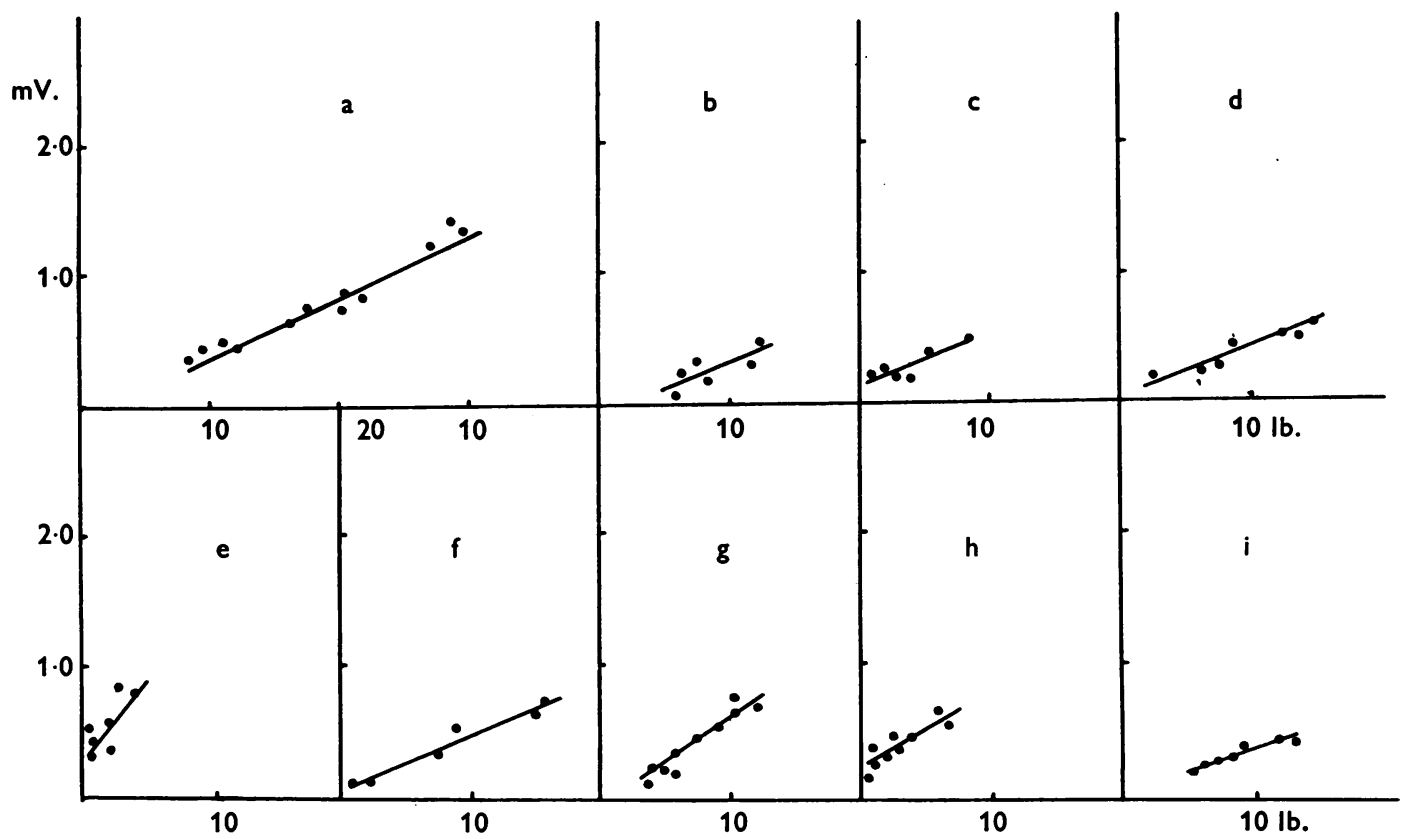

Fig. 2.-Voltage tension curves from biceps of healthy subject and eight patients with neurogenic weakness. (a) Healthy subject $\mathrm{b}=0.0521$; (b) Case 1, zoster myelitis, $\mathrm{b}=0.0358$; (c) Case 2, pseudobulbar palsy, $\mathrm{b}=0.0388$; (d) Case 3, neuralgic amyotrophy, $b=0.0427$; (e) Case 4, syringomyelia, $b=0.1077$; (f) Case 5, polyneuritis, b $=0.0442$; (g) Case 6, b = 0.0543; (h) Case 7, b = 0.0571; (i) Case 8, b = 0.2714; (g), (h), and (i) all motor neurone disease.

Curves obtained from 12 biceps muscles of 10 subjects had a slope which ranged from 0.0290 to 0.0875 with a mean regression coefficient of 0.0484 and a standard deviation of 0.0169 . With six triceps muscles of six subjects the slope was steeper, ranging from 0.0470 to 0.1553 , with a mean of 0.0972 and a standard deviation of 0.0428 .

Fig. 1 shows the effect of a prolonged fatiguing isometric contraction on the slope of the curve for one of these triceps muscles. The increased slope indicates decreased neuromuscular efficiency as defined above.

Patients with Disease of the Nervous System.These patients had muscular weakness resulting from disease of the nervous system, such as motor neurone disease, peripheral neuritis, or cerebral vascular disease. The slopes of the voltage/tension curves fell within the normal range. The high voltages associated with maximal contractions of normal muscles were not obtained because with these patients the maximal contractions were very much weaker. Over the range of tensions they were able to exert, the voltages registered were of the same order of magnitude as those of the healthy subjects. The slope of the curves for eight biceps muscles of eight subjects in this group (Fig. 2) ranged from 0.0271 to 0.1077 with a mean of 0.0510 and a standard deviation of 0.0334 . The difference between this mean and that for the 10 normal biceps muscles was not significant. The slope of the voltage/tension curve for the triceps of Case 22 (motor neurone disease) was within the normal range (Fig. 5).

Patients with Primary Muscular Disease.-Similar experiments were carried out on patients with weak muscles as a result either of a muscular dystrophy or polymyositis. Voltage/tension curves were constructed for two triceps muscles of two patients with muscular dystrophy and six biceps muscles of four patients with muscular dystrophy and two with polymyositis. The curves were of steeper slope than in the previous groups (Fig. 3) indicating decreased neuromuscular efficiency.

The regression coefficients for the triceps muscles were 0.9798 (Fig. 5) and 0.4291 . For the six biceps they ranged from 0.0723 to 0.2575 (Fig. 3) with a mean of 0.1427 and a standard deviation of 0.0694 . The significance of $t$ for the difference between the mean slope of the curves of these six muscles and of those of the 12 healthy biceps was 0.001 .

Patients with Poliomyelitis.-Nine patients who had weak muscles as a result of poliomyelitis at least one year previously were studied. The voltage/ tension curves recorded were of particularly steep slope. The regression coefficients for the seven 


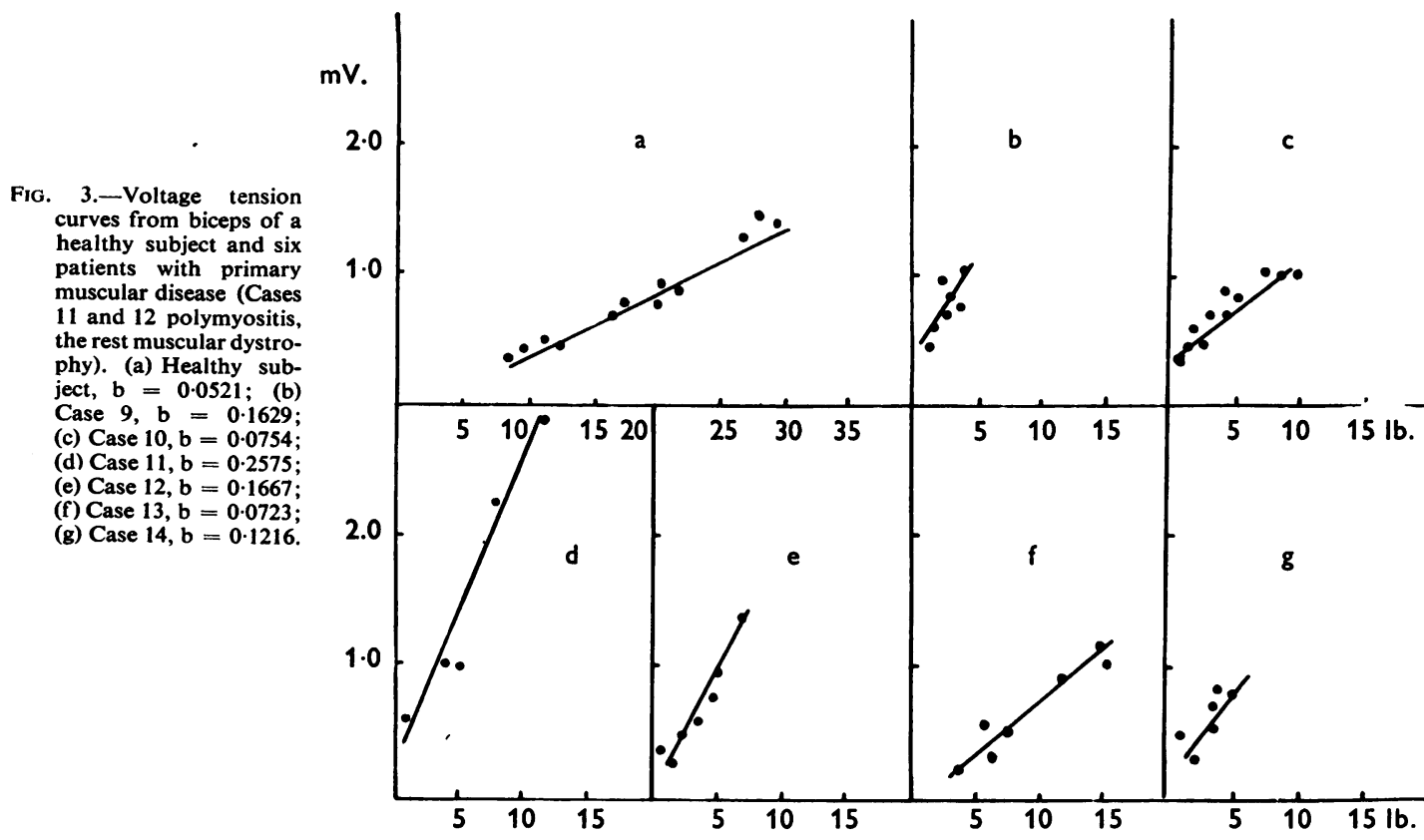

$\mathrm{mV}$.

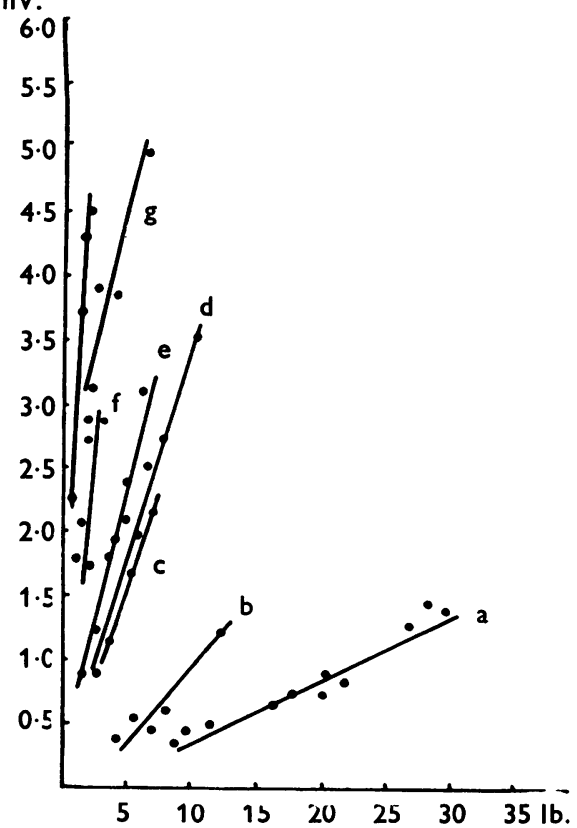

FIG. 4.-Voltage tension curves from biceps of a healthy subject and seven patients with weakness as a result of poliomyelitis. (a) Healthy subject, $b=0.0521$;

(b) Case 15, b $=0.1302$; (c) Case 16, b $=0.4430$;

(d) Case 17, b $=0.3320$; (e) Case 18, b $=0.4033$;

(f) Case 19, b = 1.0350; (g) Case 20, b = 0.4334;

(h) Case 21, b $=1 \cdot 7611$. biceps studied ranged from 0.1302 to 1.7611 with a mean of 0.6842 and a standard deviation of 0.5634 (Fig. 4). The significance of $t$ for the difference between this mean and that of the 12 normal biceps was 0.001 . The slope of the curve for the triceps of Case 24 was 0.3809 (Fig. 5) and of Case 25 was 0.2997.

The values of mean voltages associated with maximal contractions were higher in this group than in the other groups although the tensions produced were low. Thus Case 24 registered a mean voltage of $6.0 \mathrm{mV}$. during a maximal contraction of $15 \mathrm{lb}$. tension. The highest mean voltage recorded from a healthy muscle in this study was about $3.0 \mathrm{mV}$. (tension over $30 \mathrm{lb}$.).

\section{Discussion}

Bigland and Lippold (1954) in their study of the relationship between force, velocity, and integrated electrical activity in human muscles concluded that these three factors are interdependent and that the integrated electrical activity provides a measure of the number of active fibres and their frequency of activation. This is supported by the subsequent observation that in fatigued muscle the relationship between tension and integrated electrical activity is still linear but is of different slope. In a fatigued muscle more electrical activity is associated with a given tension (Edwards and Lippold, 1956). This tension is maintained by recruitment of more 


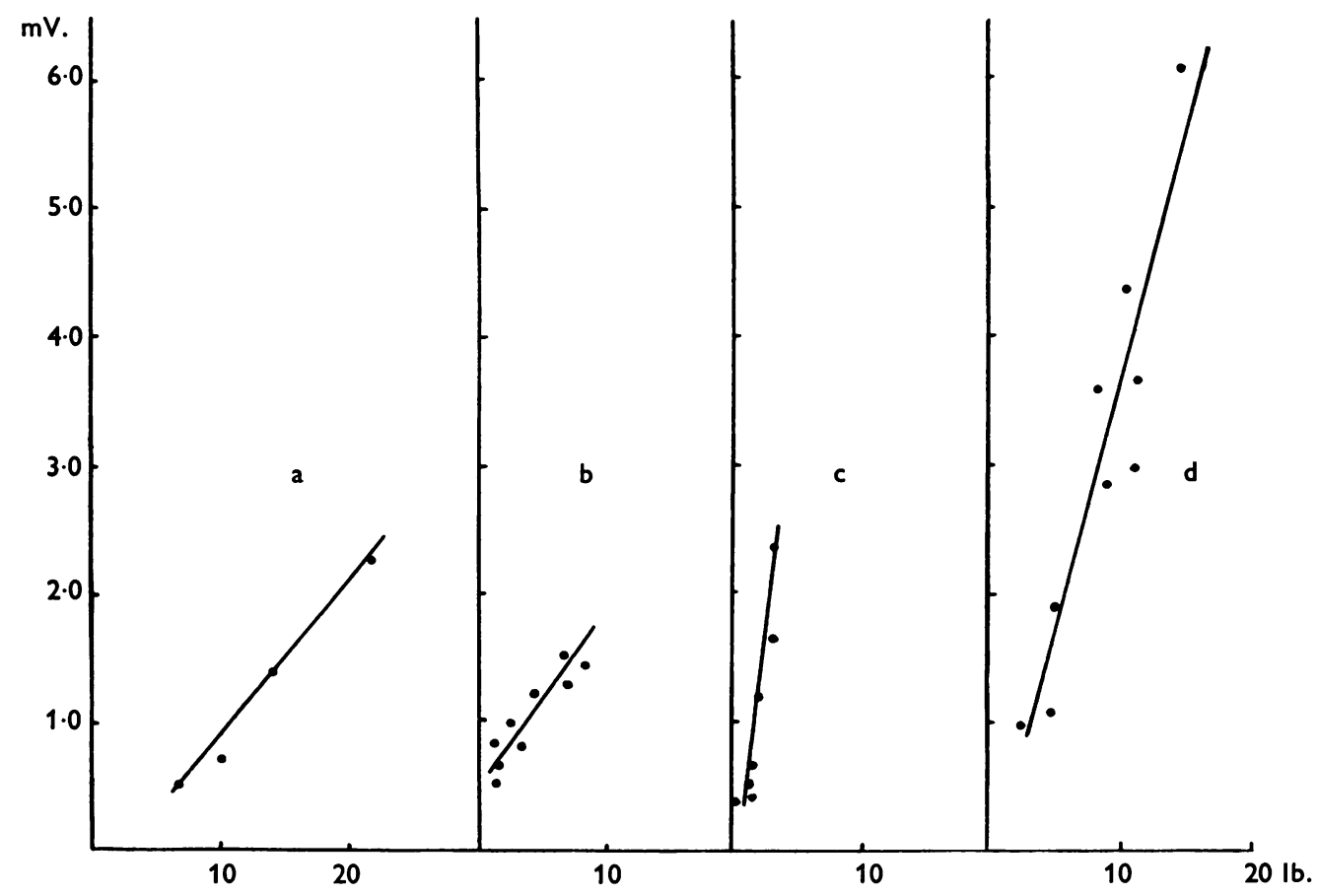

FIG. 5.-Voltage tension curves from triceps of a healthy subject and three patients with muscular weakness. (a) Healthy subject, $\mathrm{b}=0.1386$; (b) Case 22, motor neurone disease, b $=0.1292$; (c) Case 23, muscular dystrophy, b $=0.9798$; (d) Case 24, poliomyelitis, $b=0.3809$.

fibres. The slope of the curve relating voltage to tension may thus be regarded as a measure of the efficiency of the individual fibres composing a muscle (in fact the reciprocal of efficiency). The more effective the fibres as contractile units, the fewer will be required to maintain a given tension and the less steep will be the voltage/tension curve. For weak muscle the following relationship could be predicted.

If a muscle becomes weak through interference with its nerve or from an upper motor neurone lesion so that the full number of motor units can no longer be activated one would expect no alteration in the relationship between voltage and tension. The voltage and tension associated with a maximal contraction would be less in proportion to the reduction in the number of available motor units. Where weakness is due to a structural change in the muscle fibres, so that these can no longer exert their full tension, the position would be analogous to that obtaining in a fatigued muscle. To maintain a given tension, a larger number of fibres would be activated, the integrated voltage would be greater and the slope of the voltage/tension curve would be steeper.

The first group of patients reported here had muscular weakness resulting from disease of the nervous system affecting either the spinal cord or the peripheral nerves. The slopes of the voltage/ tension curves obtained from these patients lay within the normal range. This accords with the view that the loss of strength in these patients is due to a reduction in the number of available motor units.

The second group of patients had weakness resulting from disease of the muscles. Six of the eight cases had voltage/tension curves which were strikingly steeper than normal. The remaining two cases had curves with regression coefficients within the normal range though considerably greater than the mean normal value. This alteration in slope indicates that the contractile force of the muscle fibres was reduced without a corresponding reduction in the size of their action potentials (lowered mechanical efficiency). It is possible that the voltage/ tension curve may be of diagnostic value in primary muscular disorders and may also be of interest in studying the progress of the disease.

The nine patients who had weakness resulting from poliomyelitis all had curves considerably steeper than the normal and unexpectedly high voltages were associated with maximal contractions although the relationship between voltage and 
tension was still linear. All of these patients differed from the first group with disease of the nervous system in that a long period-sometimes several years - had elapsed since the disease was active. Animal experiments have demonstrated that in partially denervated muscle recovery proceeds by the surviving nerve fibres sprouting to supply denervated muscle fibres, so that the animal ends with a reduction in the number of motor units which are of greater size (Van Harreveld, 1945; Hoffman, 1950). Coërs (1954) and Wohlfart (1955) have described the same process in human muscle during recovery from poliomyelitis. If the muscles of patients in the recovering stages of poliomyelitis are examined with concentric needle electrodes it is found that the mean amplitude and the mean duration of the action potentials are greater than normal (Hertz, Madsen, and Buchthal, 1954). It is reasonable to regard these large action potentials as originating from motor units enlarged by collateral sprouting. The high mean voltages recorded here may be the result of this increase in size of the motor units. The increase in slope of the voltage/tension curves seems to suggest that motor units enlarged by collateral sprouting are less efficient contractile units, fibre for fibre, than units of physiological size.

Collateral sprouting is not a phenomenon confined to recovering poliomyelitis but is also known to occur in other conditions, notably motor neurone disease (Coërs, 1954; Wohlfart, 1957). In this condition large motor units are frequently observed on routine electromyography with needle electrodes. It is of interest therefore that the few cases of motor neurone disease studied here showed voltage/tension curves within the normal range of slope. The disease is usually rapidly progressive and it is likely that in these patients the rate of destruction of anterior horn cells was proceeding sufficiently fast to outstrip any process of recovery.

\section{Summary}

The range of slope of the curves relating integrated mean voltage to isometric tension of the upper arm muscles has been determined for a group of healthy subjects. Curves for the triceps are steeper than those obtained from the biceps.

In patients with muscular weakness due to disease of the nervous system reducing the number of available motor units the slope of the voltage/tension curves lies within the normal range.

In neuromuscular fatigue, myopathic muscle, and late cases of poliomyelitis the slope is increased, indicating reduced mechanical efficiency.

These results are as predicted from theoretical considerations except in poliomyelitis. It is suggested that this discrepancy is due to collateral sprouting of surviving neurones.

I wish to thank those physicians and surgeons in the Edinburgh hospitals who allowed their patients to be studied. Dr. J. A. Simpson provided facilities for this study and his interest, encouragement, and helpful criticism are greatly appreciated. The work was supported by a grant from the Scottish Hospitals Endowments Research Trust which is gratefully acknowledged.

\section{REFERENCES}

Bates, J. A. V., and Cooper, J. D. (1954). J. Physiol. (Lond.), 123, $28 \mathrm{P}$.

Bayer, H., and Flechtenmacher, C. (1950). Arbeitsphysiologie, 14, 261.

Bigland, B., and Lippold, O. C. J. (1954). J. Physiol. (Lond.), 123, 214.

Coërs, C. (1954). Les Variations Structurelles Normales et Pathologiques de la Jonction Neuromusculaire. Les Editions Acta Medica Belgica, Bruxelles, pp. 81-105.

Edwards, R. G., and Lippold, O. C. J. (1956). J. Physiol. (Lond.), 132,677 .

Hertz, H., Madsen, A., and Buchthal, F. (1954). J. Bone Jt Surg.,. 36-A, 902 .

Hoffman, H. (1950). Aust. J. exp. Biol. med. Sci., 28, 383.

Inman, V. T., Ralston, H. J., Saunders, J. B. de C. M., Feinstein, B., and Wright, E. W. jr. (1952). Electroenceph. clin. Neurophysiol., 4, 187.

Lenman, J. A.' R. (1959). J. Neurol. Neurosurg. Psychiat., 22, 182.

Lippold, O. C. J. (1952). J. Physiol. (Lond.), 117, 492.

Scherrer, J., Samson, M., and Paléologue, A. (1954). J. Physiol. Path. gén., 46, 887.

Van Harreveld, A. (1945). Amer. J. Physiol., 144, 477.

Wohlfart, G. (1955). Nord. Med., 54, 1075 (English summary p. 1080)_ (1957). Neurology, 7, 124. 